\title{
Effect of roughness on surface plasmons propagation along deep and shallow metallic diffraction gratings
}

\author{
Hugo Bruhier, ${ }^{1}$ Isabelle Verrier,,${ }^{1, *}$ Thiaka Gueye, ${ }^{2}$ Christelle Varenne, ${ }^{2}$ Amadou \\ Ndiaye, ${ }^{2}$ Olivier Parriaux, ${ }^{1}$ Colette Veillas, ${ }^{1}$ Stéphanie Reynaud, ${ }^{1}$ Jérôme Brunet, ${ }^{2}$ \\ AND YVES JOURLIN ${ }^{1}$ (D) \\ ${ }^{1}$ Université de Lyon, UJM, CNRS, IOGS, Laboratoire Hubert Curien, UMR CNRS 5516, 42000 Saint-Etienne, France \\ ${ }^{2}$ Université Clermont Auvergne, CNRS, Sigma Clermont, Institut Pascal, F-63000 Clermont-Ferrand, France \\ *Corresponding author: isabelle.verrier@univ-st-etienne.fr
}

Received 17 September 2021; revised 16 November 2021; accepted 29 November 2021; posted 3 December 2021; published 10 January 2022

\begin{abstract}
The roughness of shallow or deep metallic diffraction gratings modifies the propagation of surface plasmon mode along the metallic-air interface. The scattering losses lead to a spectral or angular broadening of the surface plasmon resonance (SPR) and to a shift of the resonance wavelength and coupling angle. This mechanism is deeply analyzed both experimentally and theoretically to overcome these effects when such structures, in particular deep ones, are used as SPR-based sensors.
\end{abstract}

(C) 2022 Optical Society of America under the terms of the OSA Open Access Publishing Agreement

https://doi.org/10.1364/OL.443659

Many sensors have exploited surface plasmon resonance (SPR) effects for many years [1], particularly for biological or environmental applications [2]. SPR sensors have many advantages, the main one being very good sensitivity to any changes in the refractive index of the surrounding medium, which is probed spectrally or angularly for particle/molecule detection. SPR excitation under transverse magnetic (TM) incidence is generally obtained using a prism of high refractive index or by a diffraction grating of suitable period, which enables SPR plasmon mode coupling with a non-propagative diffracted order. Losses during the plasmon propagation lead to a broadening of the resonance, thereby reducing the sensitivity of the sensor. There are many explanations for these losses, one being the quality of the surface of the metal along which the plasmon propagates, particularly its roughness. Although, in most cases, the surface roughness of shallow metallic diffraction grating does not play a major role in SPR losses, it induces changes in the propagation constants of the modes supported by plasmonic waveguides [3] and may thus strongly affect the performances of the sensor.

The objective of this study is to show the influence of roughness on plasmon resonance particularly in the case of deep sinusoidal metallic diffraction gratings and to suggest ways to counteract these effects. To our knowledge, this is the first such deep analysis and experimental demonstration. First, we simulate the standard SPR case excited by a shallow grating (0th order), second, we investigate the "optical switching effect" [4] that exploits both 0th and -1 st diffracted orders within deeper gratings. Third, we discuss the experimental results obtained in the case of the switching mechanism. Our conclusion highlights the importance of the grating roughness effect on the sensor response, especially in the case of deep gratings when roughness must be taken into consideration.

The phase-matching condition needed to couple the incident beam to the plasmon wave by the diffracted order $m$ of the grating is given by Eq. (1):

$$
k_{0} n \sin (\theta)+m K_{G}= \pm \beta,
$$

where $\beta$ is the propagation constant of the plasmon mode, and $k_{0}$ is the wavenumber of the TM incident beam under angle $\theta$ coming from a dielectric medium of refractive index $n$, which impinges on the metallic grating of period $\Lambda$ and vector $K_{G}=2 \pi / \Lambda$. The roughness of the metallic surface will change the propagation constant $\beta$ of the plasmon and hence modify the coupling conditions in terms of angle and/or wavelength. The quantity $\zeta$, hereafter called the roughness ratio, quantifies how rough the grating surface is compared to its depth. Quantity $\zeta$ is equal to the $R_{a}$ (arithmetical mean deviation compared to the smooth surface) roughness parameter divided by the peak-topeak sinusoidal grating depth $d\left(\zeta=R_{a} / d\right)$ and is introduced in the modeling. The grating roughness is modeled in two steps: 1) decomposition by Fourier expansion of a rough surface is simulated using a random Gaussian noise and 2) implementation of these complex Fourier coefficients in the software [5], which is based on Chandezon's method [6] that decomposes the grating surface profile in the same way. The Gaussian noise is simulated with 1000 points distributed randomly on either side of the grating interface with a standard deviation of $1 \mathrm{~nm}$. It is then smoothed along the grating surface by a continuous Gaussian sliding window of adapted size, which allows obtaining a correlated noise for a given roughness. This noise, compatible 


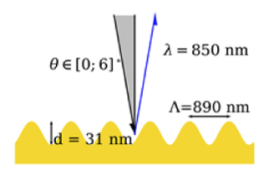

(b)

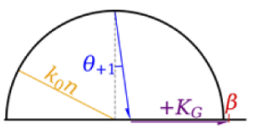

(c)

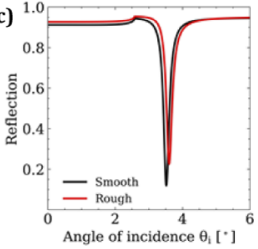

Fig. 1. (a) Scheme of the shallow sinusoidal gold grating $(\Lambda=890 \mathrm{~nm}, d=31 \mathrm{~nm})$ under a TM incident beam at $\lambda=850 \mathrm{~nm}$ in air. (b) An Ewald sphere represents coupling by the +1 st diffraction order to the propagative plasmon mode. (c) Angular responses for smooth (from left, first) and rough (from left, second $R_{a}=2.7 \mathrm{~nm}$ ) gratings.

Table 1. Effective Index for the Shallow Gold Grating $(\Lambda=890 \mathrm{~nm}, \mathrm{~d}=31 \mathrm{~nm})$

\begin{tabular}{lccc}
\hline Case & Order & Resonance & $\boldsymbol{n}_{\boldsymbol{p}}$ \\
\hline Smooth $(\lambda=850 \mathrm{~nm})$ & +1 & $3.520^{\circ}$ & 1.0164 \\
Rough $(\lambda=850 \mathrm{~nm})$ & +1 & $3.603^{\circ}$ & 1.0179 \\
Smooth $\left(\theta=0^{\circ}\right)$ & $-1,+1$ & $902.6 \mathrm{~nm}$ & 1.0141 \\
Rough $\left(\theta=0^{\circ}\right)$ & -1 & $906.15 \mathrm{~nm}$ & 1.0181 \\
& +1 & $901.35 \mathrm{~nm}$ & 1.0127 \\
\hline
\end{tabular}

with the process of the software, is added to the profile of the grating surface.

The first structure studied [Fig. 1(a)] is a one-dimensional (1D) shallow sinusoidal gold grating $(\Lambda=890 \mathrm{~nm}, d=31 \mathrm{~nm})$. SPR coupling by the +1 st diffracted order of the grating to the propagative plasmon mode is represented in Fig. 1(b) by the Ewald sphere. This geometric construction in the reciprocal space (spatial frequency) makes it possible to determine the relationship between the wavenumber $k_{0}$ of the incident beam with the vector $K_{G}$ of the grating and the constant $\beta$ of the plasmon, which propagates in the direction perpendicular to the grating lines at its interface. Intensity reflection of the 0th order versus the incidence beam angle from $0^{\circ}$ to $6^{\circ}$ under TM polarization at wavelength $\lambda=850 \mathrm{~nm}$ is presented in Fig. 1(c) for both the smooth grating and for the rough grating with the roughness parameters $R_{a}=2.7 \mathrm{~nm}$ and $\zeta=9 \%$. These roughness values come from experimental data and correspond to gratings manufactured using the standard process (lithography and etching) with $R_{a}<10 \mathrm{~nm}$. A smooth grating corresponds to a perfect sinusoidal one with no roughness $(\zeta=0 \%)$, which is generally used in the grating code. Roughness causes a small shift in resonance $\Delta \theta=0.08^{\circ}$ to a higher angle. Each resonance angle is obtained by a quadratic polynomial fit of the curve around the resonance position. Assuming quasi-lossless propagation, the corresponding approximated mode effective index is calculated by $n_{p}=|\beta| / k_{0}$, whose values are listed in Table 1 using Eq. (1) considering the +1 st coupling diffracted order.

To go further, the spectral responses of the smooth and rough shallow sinusoidal gold gratings $(\Lambda=890 \mathrm{~nm}, d=31 \mathrm{~nm})$ were also studied in the wavelength range from 880 to $920 \mathrm{~nm}$ under TM normal incidence [Fig. 2(a)]. As shown schematically in Fig. 2(b) in the reciprocal space, for an optimized sub-wavelength grating, the two propagative plasmon modes are simultaneously excited by the +1 st and the -1 st grating orders. Figure 2(c) presents the intensity reflection of the 0th order of the smooth and rough gratings with the same roughness parameter $R_{a}$ as previously. In the case of the rough grating, the (a)

(b)
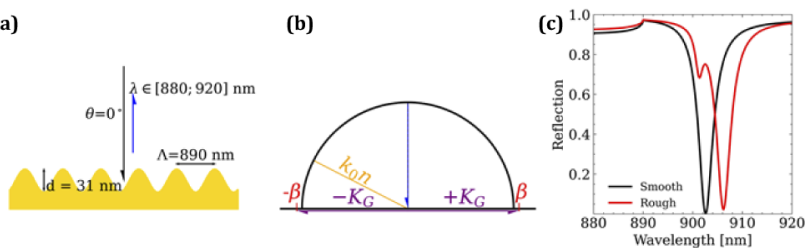

Fig. 2. (a) Scheme of the shallow sinusoidal gold grating $(\Lambda=890 \mathrm{~nm}, d=31 \mathrm{~nm})$ under a TM normal incident beam. (b) Ewald sphere represents coupling by the $(-1 \mathrm{st})+1$ st diffraction order to the two propagative plasmon modes. (c) Spectral responses for smooth (from left, first) and rough (from left, second $R_{a}=2.7 \mathrm{~nm}$ ) grating. (a)

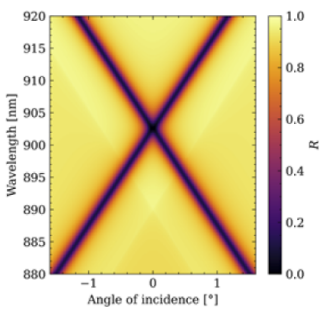

(b)

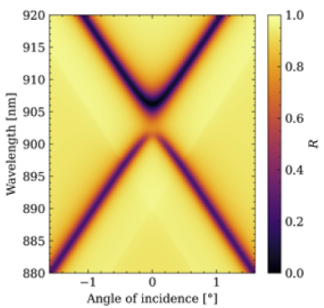

Fig. 3. Two-dimensional plot of the reflected 0th order TM intensity versus incidence angle and wavelength for the shallow sinusoidal grating $(\Lambda=890 \mathrm{~nm}, d=31 \mathrm{~nm})$ : (a) smooth; (b) rough $\left(R_{a}=2.7 \mathrm{~nm}\right)$.

plasmon resonance curve splits into two different dips separated by $\Delta \lambda=4.8 \mathrm{~nm}$, corresponding to two different propagation constants or effective indices (Table 1 ) of the two propagative modes. Therefore, when excited under TM normal incidence, the two degenerate plasmon modes propagate at two distinct speeds on a rough grating. This is illustrated by Fig. 3 which represents in false colors the intensity values of the reflected 0 th order versus the incidence angle and the wavelength: under normal incidence, two distinct wavelengths $\lambda_{1 R}=901.35 \mathrm{~nm}$ and $\lambda_{2 R}=906.15 \mathrm{~nm}$ lead to a resonance for the rough grating, whereas the smooth grating induces only one resonance centered at $\lambda_{1 S}=902.6 \mathrm{~nm}$ between $\lambda_{1 R}$ and $\lambda_{2 R}$.

The following part of the study focuses on the optical switching phenomenon, which only occurs with deep gratings [4]. It consists in the transfer of energy between propagative diffracted orders (usually between the 0th and the -1 st ones) with deep gratings and is based on the coupling of light from the evanescent diffracted orders and propagative plasmon modes. The so-called switching effect was demonstrated experimentally [4] before being investigated theoretically [7]. The shapes of the -1 st order diffraction efficiencies were first presented in [8] without studying the plasmon resonance behavior. Furthermore a few years ago, thanks to a coupled mode formalism [7], strong coupling was shown to occur between the forward-propagative and backward-propagative plasmon modes compared to the case of a shallow grating.

As previously shown in the case of a shallow grating, roughness modifies the propagation of the plasmon modes and one could also expect a change in the switching effect in the case of a deep grating. This possible change is illustrated by the following example considering a 1D sinusoidal gold grating of period $\Lambda=890 \mathrm{~nm}$ and depth $d=215 \mathrm{~nm}$ [Fig. 4(a)]. The influence of grating roughness on the switching effect was investigated under TM angular interrogation at wavelength $\lambda=850 \mathrm{~nm}$. In Fig. 4(b), 
(a)

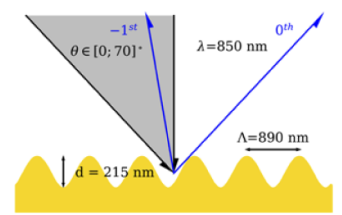

(c)

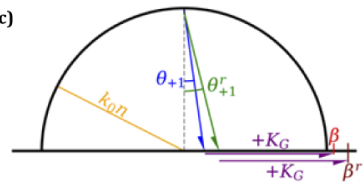

(b)

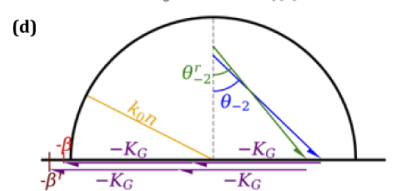

Fig. 4. (a) Scheme of the deep sinusoidal gold grating $(\Lambda=890 \mathrm{~nm}, d=215 \mathrm{~nm})$ under a TM incident beam at $\lambda=850 \mathrm{~nm}$. (b) Simulated angular responses of the switching effect: -1 st (upper curves) and 0th (lower curves) order diffraction efficiencies for the smooth (solid lines) and for the rough grating (dashed lines $\zeta=1.4 \%$ ). (c) and (d) An Ewald sphere represents coupling by the $(-2 \mathrm{nd})+1$ st order to the (backward) forward-propagative plasmon mode of constant $\beta$ (smooth case, $\Theta_{+1}$ and $\Theta_{-2}$ ) and $\beta^{\mathrm{r}}$ (rough case, $\Theta_{+1}^{\mathrm{r}}$ and $\Theta_{-2}^{\mathrm{r}}$ ).

Table 2. Effective Indices at the Two Resonances for the Deep Grating $(\Lambda=890 \mathrm{~nm}, d=215 \mathrm{~nm})$ at $\lambda=850 \mathrm{~nm}$

\begin{tabular}{llccc}
\hline Case & Resonance & Order & Angle $\left[{ }^{\circ}\right.$ ] & $\boldsymbol{n}_{\boldsymbol{p}}$ \\
\hline \multirow{2}{*}{ Smooth } & First & +1 & 4.4 & 1.0318 \\
& Second & -2 & 61.5 & 1.0314 \\
Rough & First & +1 & 5.4 & 1.0492 \\
$\zeta=1.4 \%$ & Second & -2 & 59.5 & 1.0485 \\
Rough & First & +1 & 8 & 1.0942 \\
$\zeta=2.6 \%$ & Second & -2 & 54.4 & 1.0970 \\
\multirow{2}{*}{ Experience } & First & +1 & $8.2 \pm 0.5$ & $1.093 \pm 0.009$ \\
& Second & -2 & $54.8 \pm 0.5$ & $1.097 \pm 0.005$ \\
\hline
\end{tabular}

the simulated 0th and -1 st order diffraction efficiencies of the smooth grating (solid lines) are compared with those of a grating with a roughness coefficient $R_{a}=3 \mathrm{~nm}$, which is very low compared with its depth and corresponds to a ratio $\zeta=1.4 \%$ (dashed lines). The first minima of the -1 st order at $\theta=4.4^{\circ}$ (smooth) and $\theta=5.4^{\circ}$ (rough) correspond to the coupling by the +1 st diffractive order to the forward-propagative plasmon mode. For the second minima at the incidence angles $\theta=61.5^{\circ}$ (smooth) and $\theta=59.5^{\circ}$ (rough), the backward-propagative plasmon mode is excited by the -2 nd diffractive order.

The numerical results of the resonance angles and effective index computation retrieved by Eq. (1) are summarized in Table 2 and show a marked change in the modes effective indices even with a very low roughness ratio $(\zeta=1.4 \%)$, and hence major shifts in the angular resonance positions $\left(\Delta \theta_{1}=1^{\circ}\right.$ and $\Delta \theta_{2}=2^{\circ}$ ) compared with the minor shift in the case of a shallow grating $\left(\Delta \theta=0.08^{\circ}\right)$ as illustrated in Fig. 1. This analysis reveals on the one hand that the coupling angle of the forwardpropagative plasmon by the +1 st order increases in the presence of roughness, as previously shown with the shallow grating, whereas the coupling angle of the backward-propagative plasmon by the -2 nd order becomes smaller. This is explained by the Ewald sphere [Figs. 4(c)-4(d)] that illustrates the result of the increase in the propagation constants from $|\beta|$ to $\left|\beta^{r}\right|$ in the presence of roughness and the change in the coupling conditions
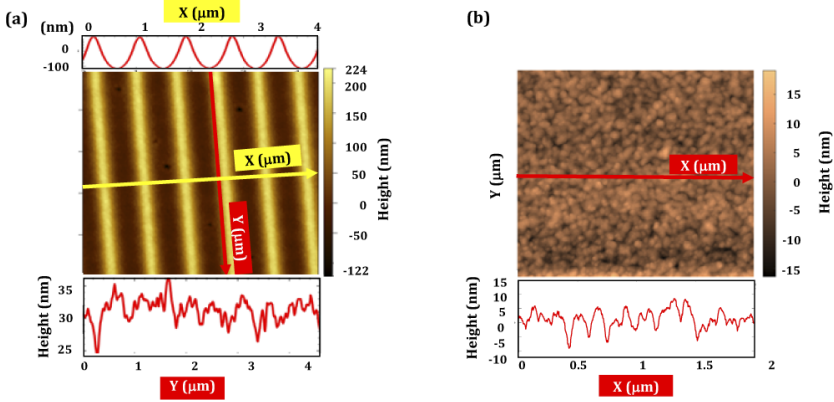

Fig. 5. AFM scans and profiles: (a) at the edge of the line of the deep gold grating $(\Lambda=890 \mathrm{~nm}, d=215 \mathrm{~nm})$; (b) on the flat surface.

at both resonances. The coupling angles of both modes become closer in the presence of roughness [Fig. 4(b)]. On the other hand, as shown previously in the case of shallow metallic grating (Fig. 3), under normal incidence, the inter-mode coupling results in a gap between the theoretical coupling conditions [Eq. (1)] and the real resonance positions. In the case of plasmon switching, grating roughness can strengthen inter-plasmon coupling and modify the resonance positions much more than in the case of plasmon coupling with shallow gratings, where inter-mode coupling occurs only under normal incidence.

To check these simulations and confirm our hypotheses, measurements were taken using a grating produced on a glass substrate on which the resist was deposited, exposed by photolithography under UV light, and revealed in a developer. The gold layer was deposited using a thermal evaporation technique in high vacuum at a rate of $1 \AA /$ s controlled by a quartz crystal monitor while the temperature of the sample was maintained at $85^{\circ} \mathrm{C}$. The gold layer was approximately $120 \mathrm{~nm}$ thick, to consider an infinite metallic layer and thus to avoid the influence of the photoresist layer and the substrate. Atomic force microscopy (AFM) [Fig. 5(a)] makes it possible to check the shape of the grating and to estimate the experimental roughness coefficient $R_{a} \approx 2 \pm 1 \mathrm{~nm}$ using the profile in the Y direction. On the same unstructured sample, the experimental roughness coefficient is higher: $R_{a} \approx 2 \pm 1 \mathrm{~nm}$ [Fig. 5(b)]. At such low roughness values $(\zeta \approx 1 \%)$, AFM measurements reach their limits and the roughness value measured on the flat surface is the one considered in the following. The diffraction efficiencies measurement setup was conceived so to achieve an angular interrogation of the grating [Fig. 6(a)]. A $1 \mathrm{~mW}$ laser diode emitting at wavelength $\lambda=850 \mathrm{~nm}$ was used as a light source under TM polarization. An (a)

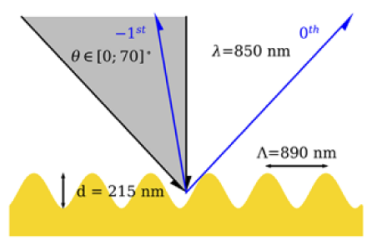

(b)

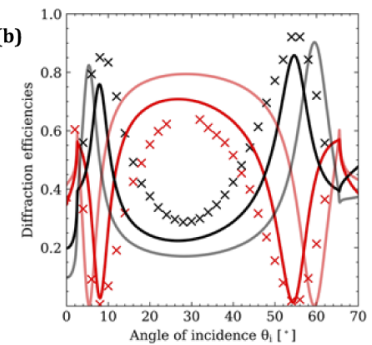

Fig. 6. (a) Scheme of the deep sinusoidal gold grating $(\Lambda=890 \mathrm{~nm}, d=215 \mathrm{~nm})$ under a TM incident beam at $\lambda=850 \mathrm{~nm}$. (b) Measured efficiencies (crosses) of the -1 st order (maximum in the middle) and 0th reflected order (minimum in the middle) versus incidence angle compared with simulations (solid lines), where $\zeta=1.4 \%$ (outer curves) and $\zeta=2.6 \%$ (inner curves). 
(a)

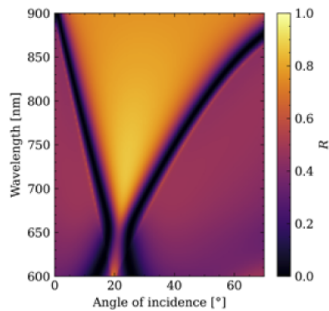

(b)

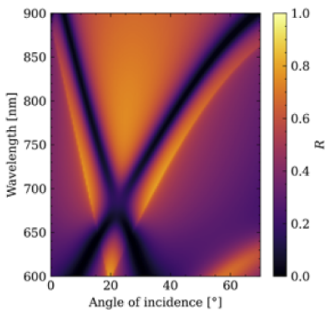

Fig. 7. Two-dimensional plot of the diffracted -1 st order TM intensity versus incidence angle and wavelength for the deep grating $(\Lambda=890 \mathrm{~nm}, d=215 \mathrm{~nm})$ : (a) smooth; (b) rough $(\zeta=2.6 \%)$.

IR photodiode was connected to a homemade transimpedance circuit, and an NI 9215 module plugged into a cDAQ-9174 rack and connected to a computer to measure the photodiode output voltage. The measurements (crosses) of the -1 st and the 0th order diffraction efficiencies are plotted in Fig. 6(b) versus the incidence angle controlled by a goniometer. Diffraction efficiencies were likewise simulated using a roughness ratio $\zeta=1.4 \%\left(R_{a}=3 \mathrm{~nm}\right)$, whose value was previously obtained by AFM measurement (outer solid curves) and can also be determined by adjusting the positions of the theoretical resonance angles with respect to the experimental ones (inner solid curves) leading to a roughness ratio of $\zeta=2.6 \%\left(R_{a}=5.7 \mathrm{~nm}\right)$. This last value was found manually by trial and error and was higher than that measured by AFM. As a matter of fact, the $\zeta$ value obtained from the fit is also subject to calculation errors and uncertainties in the measurement of the resonance angles; furthermore, the value includes all the imperfections of the grating, which is not uniform over the whole probed area. That is why, despite the same tendency and the small difference in roughness, the theoretical and experimental curves differ in amplitudes and the resonance angles do not match. Furthermore, both resonances broadened and shifted with an increase in roughness.

Table 2 presents the effective indices computed according to Eq. (1) using the experimental values of the two angular resonances and those obtained by the fit; and confirms the difference between the propagation constants of the forward- and backward-propagative modes. Figure 7 represents (in false colors) the -1 st order diffraction efficiencies versus the incidence angle and the wavelength for the smooth and the rough deep gratings. At wavelength $\lambda=650 \mathrm{~nm}$, two resonances around the angle of $\theta=21^{\circ}$ appear in the case of the smooth grating [Fig. 7(a)] while only one resonance at $\theta=21^{\circ}$ appears at wavelength $\lambda=675 \mathrm{~nm}$ in the case of the rough grating [Fig. 7(b)].

The same curves can be plotted for the 0th order where minima efficiency regions become the maxima ones.

This Letter highlights the effect of roughness on plasmon resonances in the case of deep and shallow diffraction gratings. Particularly in the case of deep gratings (above hundreds of $\mathrm{nm}$ for subwavelength gratings), inter-plasmon coupling is strengthened by roughness that induces significant shifts in resonance as well as broadening. When deep grating is used as a sensor, the operating points are located at positions corresponding to equal diffraction efficiencies of the 0th and the -1 st orders. The slopes of the efficiency curves at these points determine the sensitivity of the sensor, which will therefore be affected by a change caused by roughness of the diffraction grating. However, even if the losses arising from roughness (scattering, depolarization, etc.) cannot be completely offset, maintaining resonance at a fixed wavelength or angle is possible by modifying the design of the grating using rigorous modeling taking the real roughness of the grating into consideration.

Funding. Agence Nationale de la Recherche (ANR CAPTAIN project (ANR-18-CE04-008)).

Disclosures. The authors declare no conflicts of interest.

Data availability. Data underlying the results presented in this Letter are not publicly available at this time but can be obtained from the authors upon reasonable request.

\section{REFERENCES}

1. S.A. Maier, Plasmonics Fundamentals and Applications (Springer, 2007).

2. J. Homola, Chem. Rev. 108, 462 (2008).

3. T. Shibata, H. Ikeda, H. Nishiyama, K. Tawa, and J. Nishii, Phys. Procedia 48, 179 (2013).

4. J. Sauvage-Vincent, Y. Jourlin, V. Petiton, A. V. Tishchenko, I. Verrier, and O. Parriaux, Opt. Express 22, 13314 (2014).

5. N. Lyndin and B. Usievich, MC Grating software (2013).

6. J. Chandezon, G. Raoult, and D. Maystre, J. Opt. 11, 235 (1980).

7. A. V. Tishchenko and O. Parriaux, IEEE Photonics J. 7, 1 (2015).

8. M. C. Hutley, Optica Acta: Int. J. Opt. 20, 607 (1973). 